\title{
Prostate cancer boost using high-dose-rate brachytherapy: early toxicity analysis of 3 different fractionation schemes
}

\author{
Hussam Hijazi, MD',2, Daniel Chevallier, MD³, Jocelyn Gal, MSc4, Marie-Eve Chand, MD!, Mathieu Gautier, MSc', \\ Jean-Michel Hannoun-Levi, MD, PhDl \\ 'Department of Radiotherapy, Antoine Lacassagne Cancer Center, University of Nice-Sophia, Nice, France, ${ }^{2}$ Department of Radiotherapy. \\ King Abdulaziz University Hospital, Jeddah, Saudi Arabia, ${ }^{3}$ Urology Department, Pasteur Academic Hospital, Nice, ${ }^{4}$ Statistic Unit, Antoine \\ Lacassagne Cancer Center, Nice, France
}

\begin{abstract}
Purpose: To analyse early toxicity of high-dose-rate brachytherapy (HDRB) boost for prostate cancer using 3 fractionation schemes.

Material and methods: From February 2009 to May 2012, after the first course of external beam radiation therapy (EBRT 46 Gy/23 f), 124 patients underwent HDRB boost for low (7\%), intermediate (19\%), and high-risk (73\%) prostate cancers. From February to December 2009, Group 1 (G1) = 18 Gy/3 f/2 d (24\%); from January 2010 to April 2011, Group 2 (G2) = 18 Gy/2 f/2 d (42\%), and from May to September 2011, Group 3 (G3) = 14 Gy/1 f/1 d (34\%). Planning and CT-scan was performed before each fraction. Dose constraints for G1/G2 were $V_{100}$ rectum $=0$ and $V_{125}$ urethra $=0$, while for $\mathrm{G} 3 \mathrm{~V}_{90}$ rectum $=0$ and $\mathrm{V}_{115}$ urethra $=0$. Genito-urinary (GU) and Gastro-intestinal (GI) acute toxicities were assessed at 1 month (for the 3 fractionation schemes) and 6 months (for $18 \mathrm{~Gy} / 3 \mathrm{f}$ and $18 \mathrm{~Gy} / 2 \mathrm{f}$ ) after the boost (CTCv3.0).

Results: Median follow-up was 25 months (8-46.9), median age was 71 years (50-82), and median CTV was 31 cC (16-71). The grades of acute GI and GU toxicities at 1 and 6 months after HDRB were mainly Grade 1 with few Grade 2 (GU: 5\% at 1 month; GI: $1 \%$ at 6 months). One patient developed G4 sepsis toxicity 2 days after HDRB and recovered without after-effects. No significant differences were observed at 1 and 6 months after the HDRB between treatment groups.

Conclusions: The right fractionation remains under discussion, but prostate cancer HDRB boost using a single fraction (providing similar results in terms of acute toxicity) is more comfortable for the patient, and less time consuming for the medical staff.

Key words: boost, early toxicity, fractionation, high-dose-rate brachytherapy, prostate cancer.

\section{Purpose}

Aside from non-melanoma skin cancer, prostate cancer (PC) is the most common cancer in men in the United States and Europe, with a rapid increasing incidence in the last two decades [1]. Radiotherapy is one of the most important curative options in treating localized PC. For high-risk PC, standard dose (70 Gy) plus long-term anti-androgen therapy became the gold standard after the publication of EORTC 22863 [2] and RTOG 92-02 [3] trial results. Furthermore, Pollack et al. [4] shown that dose escalation (78 Gy) was a key point for biochemical control. Many studies have confirmed positive effect of dose escalation on disease local control using radiation therapy [5-8]. However, dose escalation delivered through 3D ex- ternal beam radiation therapy (EBRT) was associated with a higher rate of gastro-intestinal (GI) and genito-urinary (GU) toxicities [9]. Using intensity modulated radiation therapy (IMRT), Zelefsky et al. [10] shown that a total dose of 81 Gy could be delivered to the prostate increasing the rate of biochemical control without increasing the rate of GU and GI toxicities. Intensity-modulated radiation therapy for PC is generally performed through 8 to 9 consecutive weeks; nevertheless, due to the low $\alpha / \beta$ ratio of the prostatic tissue, clinical research was directed toward hypofractionation concept. Randomized trials comparing standard radiation dose (2 Gy/f, $1.8 \mathrm{~Gy} / \mathrm{f}$ ) versus "soft" hypofractionated regimen (2.5 Gy/f to $3 \mathrm{~Gy} / \mathrm{f}$ ) using EBRT confirmed that the two regimens were equivalent in terms 
of acute and late toxicity [11,12]. High-dose rate (HDR) brachytherapy boost following EBRT delivering a total dose of 46 Gy to the pelvis or prostatic fossa, represents a smart technique to not only increase the dose to the prostate, but also significantly reduce the number of round trips to the radiation facility.

In February 2009, we integrated the HDR brachytherapy boost program in PC treatment. We used 3 different fractionation schemes consecutively. We report the results in terms of acute GU and GI toxicity observed between these three different regimens.

\section{Material and methods \\ Patient characteristics}

Between February 2009 and July 2013, 216 consecutive patients underwent a HDR brachytherapy boost for PC. Over the study period, two different treatment planning systems (TPS) were used: Plato ${ }^{\mathrm{TM}}$ (Nucletron BV, Veenendaal, The Netherlands) from February 2009 to May 2012, and Oncentra Brachy ${ }^{\mathrm{TM}}$ (Nucletron, an Elekta company, Elekta AB, Stockholm, Sweden) from June 2012 until now. In order to avoid any comparative bias, we analysed the first 124 patients treated with the same TPS (Plato ${ }^{\mathrm{TM}}$ ). From 02/2009 to 01/2010, 30 patients (Group $1=$ G1;24\%) were treated with $3 \times 6$ Gy over 2 days. In order to make this procedure more comfortable for the patient and less time-consuming for the medical staff, from 01/2010 to 01/2011, 52 patients (Group 2 = G2; $42 \%$ ) received 2 fractions of 9 Gy over 2 days, while from 01/2011 to 05/2012, 42 patients (Group $3=$ G3; 34\%) were treated with a single fraction of $14 \mathrm{~Gy}$. This observational study retrospectively analysed the acute GU and GI toxicities occurred during the first 6 months after the implant in the 3 treatment groups. The 3 different fractionation regimens were approved by the institutional review board.

All the patients presented with a histologically proved non-metastatic PC. All the patients underwent imaging studies, including magnetic resonance, computed tomography, and bone scan. Appropriate patient selection was performed according to the American Brachytherapy Society (ABS) consensus guidelines for HDR prostate brachytherapy [13].

\section{Irradiation treatment techniques and planning}

Patients were treated with a first course of EBRT, delivering 46 Gy in 23 fractions. 3D conformal EBRT was used for achieving this first treatment step. The dose was delivered to the ICRU point using high-energy $\mathrm{X}$-photons (>10 MV). According to the calculated risk of lymph node involvement, the clinical target volume (CTV) was the whole pelvis or the prostatic fossa (prostate and seminal vesicles) for high- ( $\geq 15 \%)$ and low-risk $(<15 \%)$ of lymph node involvement, respectively [14]. The planning target volume (PTV) was defined as a $1 \mathrm{~cm}$ margin around the CTV in all directions, and reduced to $5 \mathrm{~mm}$ at the prostate rectal interface. Short and long-term androgen deprivation therapy (ADT) was proposed to intermediate and high-risk patients, respectively.
After completion of the EBRT, patients underwent HDR brachytherapy boost. This procedure was performed under general anaesthesia. Using TransRectal UltraSound (TRUS) guidance, 15 to 17 catheters (Sharp Needles ${ }^{\mathrm{TM}}$; Nucletron, an Elekta company, Elekta AB, Stockholm, Sweden) were implanted transperineally and periurethrally through a dedicated template. After recovery, post-implant planning CT-scan was directly performed in the radiation oncology department for treatment planning purposes. CTV (prostate) and organs at risk (OAR - urethra and rectum) were outlined [15]. The prostatic portion of the urethra was outlined plus a $10 \mathrm{~mm}$ additional margin above the base and below the apex, while the rectum was outlined from the anorectal junction to the rectosigmoid junction. Brachytherapy boost delivered $3 \times 6 \mathrm{~Gy}, 2 \times 9 \mathrm{~Gy}$, and $1 \times$ 14 Gy for G1, G2, and G3, respectively. Considering $\alpha / \beta$ $1.5 \mathrm{~Gy}$ for prostatic tissue, EQD2 (equivalent dose at $2 \mathrm{~Gy} /$ fraction) were $39 \mathrm{~Gy}, 54 \mathrm{~Gy}$, and 62 Gy for G1, G2, and G3, respectively [16]. Dose constraints for prostate were $V_{100}$ (CTV receiving $100 \%$ of the prescribed dose) $>95 \%, \mathrm{~V}_{150}$ $<35 \%$, and $\mathrm{V}_{200}<15 \%, \mathrm{D}_{90}$ (dose delivered to $90 \%$ of $\mathrm{CTV}$ ) $>105 \%$, and $\mathrm{D}_{100} \%>80 \%$. Dose constraints used for OARs relied on the brachytherapy regimen groups. Because of the slight dose escalation between G1/G2 (EQD2 ${ }_{\alpha \beta 1.5}$ $39 \mathrm{~Gy}, 54 \mathrm{~Gy})$ and $\mathrm{G} 3\left(\mathrm{EQD} 2_{\alpha \beta 1.5} 62 \mathrm{~Gy}\right)$, more restrictive dose constraints were considered for $\mathrm{G} 3$ patients. Consequently, for G1/G2, $\mathrm{Vu}_{125}$ (percentage of the urethra volume receiving $125 \%$ of the prescribed dose) and $\mathrm{Vr}_{100}$ (percentage of the rectum volume receiving 100\% of the prescribed dose) should be less than 1\%, while for G3 patients, $\mathrm{Vu}_{115}$ (percentage of the urethra volume receiving $115 \%$ of the prescribed dose) and $\mathrm{Vr}_{90}$ (percentage of the rectum volume receiving $90 \%$ of the prescribed dose) should be less than 1\% (Fig. 1). Dose-volume adaptation was manually achieved using graphical optimization (Platoä, Nucletron BV, Veenendaal, The Netherlands) by dwell location and time variation. Patients treated with multiple fractions after a single insertion were re-scanned before each fraction, followed by re-optimization with regard to the risk of catheter migration [17]. The first fraction was given post-operatively (at day 0), while the following fractions were given at day 1 (with at least 6 hours interval between the $2^{\text {nd }}$ and $3^{\text {rd }}$ fraction for G1).

\section{Follow-up}

Patients were followed at 1 month after the implant, then every 6 months with an assessment of serum prostate specific antigen level (PSA), physical examination, patient symptom assessment, and digital rectal examination in case of rising PSA. Acute GU and GI toxicities were scored according to the Common Terminology Criteria for Adverse Event (CTCAE v3.0) [18]. For GU complications, we analysed stricture/stenosis, frequency/urgency, retention, incontinence, cystitis and bladder spasms while for GI complications we analysed haemorrhoids, anal incontinence, and proctitis. Because a longer follow-up is needed to assess sexual toxicity, and due to the high number of patients who received ADT, this complication was not assessed. Follow-up evaluation was performed alternatively by radiation oncologist and 

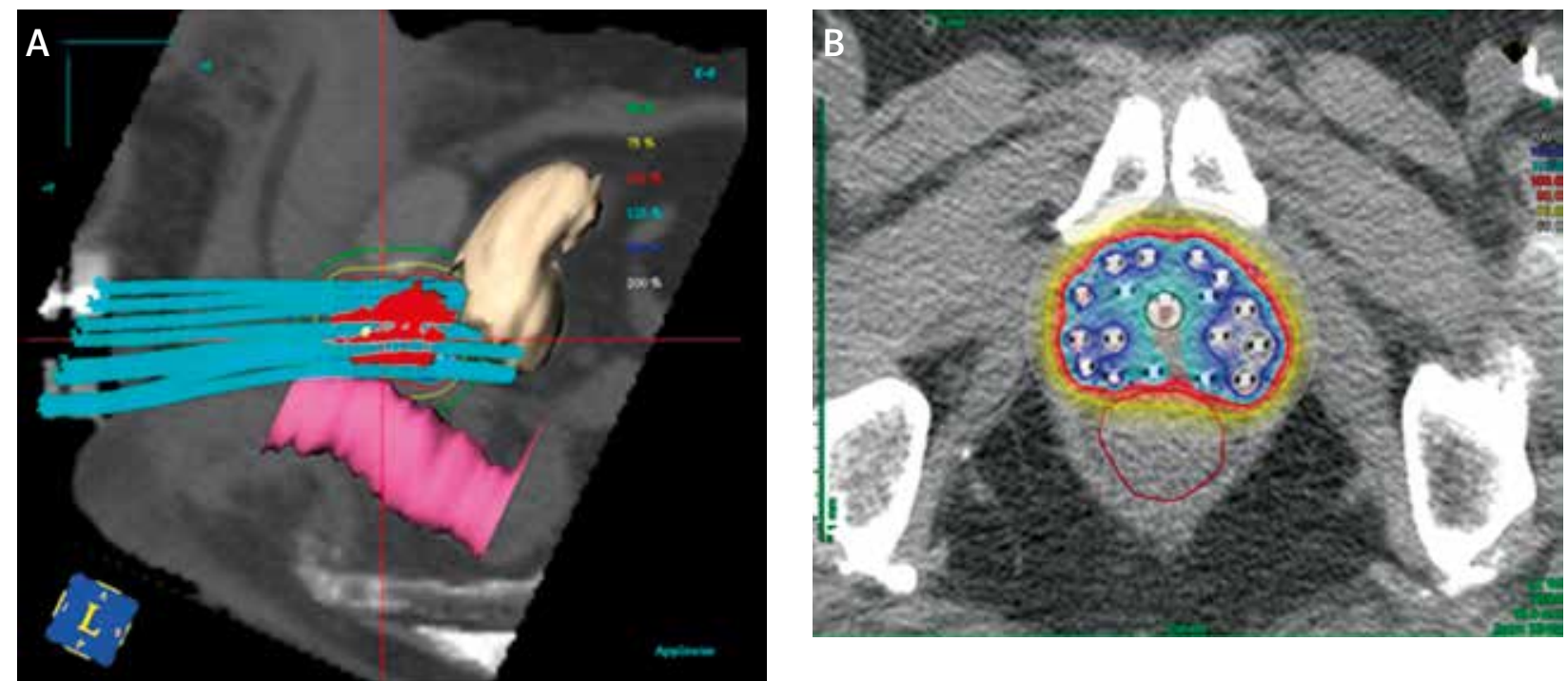

Fig. 1. Dose distribution obtained after planning using Plato (Nucletron BV, Veenendaal, The Netherlands) on sagittal (A) and transversal (B) view

urologist. Biochemical failure was defined as PSA nadir plus $2 \mathrm{ng} / \mathrm{ml}$ [19].

\section{Statistical analysis}

Data were entered and stored on an Access file, and then transferred into R.2.14.1 software for statistical analysis. Quantitative variables were summarized by means, standards deviation, medians, and range. Qualitative variables were described by counts and percentages. $\chi^{2}$ test or Fisher's exact test was used for category comparison. Student test or Wilcoxon's test was used to compare continuous variables. ANOVA or non-parametric Kruskal-Wallis was performed to test variables expressed as categories versus continuous variables. If this test was significant, we used the test of Tukey or Nemenyi test to compare these categories. We also showed the median survival and confidence intervals at $95 \%$ of the studied population (Kaplan-Meier method). Tests of significance were two-sided, and considered significant when the $p$ value was 0.05 or less.

\section{Results}

\section{Patient and treatment characteristics}

Patient and treatment characteristics are reported in Table 1. Regarding patient (age, risk group factors) and treatment (EBRT, ADT) data, no significant differences were observed between the 3 treatment groups. Median age was 69.7 years (ranged 49-82). The majority of the patients were classified as high $(73.4 \%)$ or intermediate $(19.3 \%)$ risk regarding to $\mathrm{D}^{\prime}$ Amico classification [20]. Sixty-five percent of the patients (81 patients) received whole pelvic EBRT while 80\% (99 cases) underwent ADT. The median time interval between EBRT and HDR brachytherapy boost was 14 days (ranged 0-43).

Dosimetric data are reported in Table 2. Because no significant dosimetric differences were noticed between
G1/G2 patients regarding $1^{\text {st }}, 2^{\text {nd }}$ and $3^{\text {rd }}$ post-implant CT-scan (data not shown), the $1^{\text {st }}$ CT-scan was used for dosimetric comparisons between the 3 treatment groups. Median CTV were equivalent in 3 groups $(36,33$, and 32 cc for G1, G2, and G3, respectively, $p=0.088)$. $D_{90}$ for G3 (105\%) was significantly decreased compared to the $\mathrm{D}_{90}$ calculated for G1 $(114 \%)$, and G2 $(111 \%)(p<0.001)$ as well as $V_{100}(97,96$, and $95 \%$ for G1, G2, and G3, respectively; $p=0.013$ ), while $\mathrm{V}_{150}$ and $\mathrm{V}_{200}$ significantly decreased from G1 to G3 (V $\mathrm{V}_{150}: 45,36$, and $27 \%$ for G1, $\mathrm{G} 2$, and G3, respectively; $p<0.001$ and $\mathrm{V}_{200}$ : 16, 12, and 9 for G1, G2, and G3, respectively $p<0.001$ ). Dose Homogeneity Index (DHI) for G3 $(69 \%)$ was significantly better compared to DHI noticed for G1 (54\%) and G2 (61\%) $(p<0.001)$. For OARs (urethra and rectum), dosimetric results were significantly improved in G3 compare to G1 and G2 $(p<0.001)$ (Table 2). Assuming $\alpha \beta 3$ Gy for urethra and rectum, EQD2 of the dose delivered by the boost to OARs significantly decreased from G1 to G3 for both urethra $\left(\mathrm{D}_{10 \mathrm{u}}: 52,47\right.$, and 46 Gy for $\mathrm{G} 1, \mathrm{G} 2$, and G3, respectively; $p<0.001)$ and rectum $\left(\mathrm{D}_{2 \mathrm{r}}: 28,27\right.$, and $26 \mathrm{~Gy}$ for G1, G2, and G3, respectively; $p<0.001$ ) (Table 2).

\section{Acute genito-urinary and gastro-intestinal toxicity analysis}

The median follow-up was 25 months (ranged 8-46.9). Genito-urinary and GI complication grades for each treatment group were summarized in Table 3 . The rates of acute GI and GU toxicities observed at 1 and 6 months after HDR brachytherapy boost were mainly Grade 1 with few Grade 2 (GU: 5\% at 1 month; GI: $1 \%$ at 6 months). No Grade 3 toxicity was observed in the whole population, while one patient in Group 1 developed Grade 4 GU toxicity consisting in a post-operative septic syndrome treated in intensive-care unit during 14 days. This patient recovered after 1 month without GU complications at 6 months.

Regarding the acute GU and GI toxicities occurred in the 3 treatment groups, no significant differences were 
Table 1. Patient characteristics according to the treatment group

\begin{tabular}{|c|c|c|c|c|c|c|c|}
\hline \multirow[t]{2}{*}{ Data } & \multicolumn{2}{|c|}{3 fractions (G1) } & \multicolumn{2}{|c|}{2 fractions (G2) } & \multicolumn{2}{|c|}{1 fraction (G3) } & \multirow[t]{2}{*}{$p$ value } \\
\hline & $n /$ Average & $\% /($ Min-Max) & $n /$ Average & $\% /($ Min-Max) & $n /$ Average & $\% /($ Min-Max) & \\
\hline \# Pts & 30 & 24 & 52 & 42 & 42 & 34 & \\
\hline Age & 69 & $(50-82)$ & 70 & $(59-80)$ & 68 & $(49-81)$ & 0.250 \\
\hline T stage & & & & & & & 0.103 \\
\hline T1c-2a-2b & 12 & 25 & 15 & 31 & 21 & 44 & \\
\hline$T 2 c-3 a-3 b$ & 18 & 33 & 37 & 49 & 21 & 28 & \\
\hline PSAi & 13 & $(5.35-58)$ & 21 & $(1.65-369)$ & 13 & $(3-114)$ & 0.6 \\
\hline Gleason score & & & & & & & 0.4 \\
\hline 6 & 9 & 24 & 15 & 39 & 14 & 37 & \\
\hline 7 & 8 & 17 & 22 & 48 & 16 & 35 & \\
\hline$\geq 8$ & 13 & 33 & 15 & 37 & 12 & 30 & \\
\hline Risk groups & & & & & & & 0.633 \\
\hline Low & 1 & 12 & 4 & 50 & 4 & 38 & \\
\hline Intermediate & 5 & 20 & 8 & 34 & 11 & 46 & \\
\hline High & 24 & 27 & 40 & 43 & 27 & 30 & \\
\hline Pelvic EBRT & & & & & & & 0.149 \\
\hline Yes & 16 & 20 & 38 & 47 & 27 & 33 & \\
\hline No & 14 & 33 & 14 & 33 & 15 & 34 & \\
\hline ADT & & & & & & & 0.110 \\
\hline Yes & 24 & 24 & 45 & 46 & 30 & 30 & \\
\hline No & 6 & 23 & 7 & 27 & 12 & 50 & \\
\hline Int RT/Brachy (d) & 12 & $(0-43)$ & 17 & $(1-42)$ & 14 & $(5-32)$ & 0.065 \\
\hline
\end{tabular}

$n$-number of patients, EBRT - external beam radiation therapy, ADT-androgen deprivation therapy, Int RT/Brachy - time interval between the end of EBRT and high-dose rate brachytherapy expressed in days

observed at 1 and 6 months after the HDR brachytherapy boost between G1, G2, and G3 treatment groups (Table 3).

\section{Discussion}

Dose escalation for prostate cancer radiation treatment represents a key point in order to improve biochemical control. According to the meta-analysis published by Viani et al. [21], high-dose radiotherapy was superior to conventional dose radiotherapy in preventing biochemical failure in all risk groups, suggesting that high-dose radiotherapy should be offered to all patients regardless of their risk status. The low $\alpha / \beta$ ratio indicates that prostate tumours are more sensitive to high radiation doses per fraction than most other malignancies [16]. Therefore, many investigators have directed their efforts to deliver hypofractionated EBRT. In this field, the use of brachytherapy boost in addition to pelvic EBRT up to 46 Gy represents a smart and attractive option [22,23]. Indeed, brachytherapy allows an "intrinsic" dose escalation due to the overdose volumes generated by the interstitial position of the radioactive source within the prostatic tissues, and lead to a biological advantage over external beam radiation boost technique [24]. Brachytherapy boost has not only a potential advantage on clinical outcome, but also allows to make the treatment more acceptable for the patient due to less transportations to the medical insurance for reimbursement, and for the medical staff dealing with a less time consuming procedure. In this field, HDRB appears to give at least equivalent clinical outcomes compared to low-dose rate brachytherapy [25].

A matched-pair analysis comparing prostate cancer EBRT + HDR vs. EBRT alone demonstrated a significant advantage in 5-year biochemical control in favour to the combination modality [26]. Two randomized trials have confirmed the superiority of the combination therapy using either low [27] or high-dose rate [28] iridium sources in terms of biochemical control with at least similar late GU and GI toxicity with a potential advantage for acute rectal complications [29]. However, these studies used as "conventional" EBRT arm a total dose around 70 Gy which is now considered as a low dose regarding biochemical control probability. Nevertheless, even in case of very high-dose delivered to the prostate (86.4 Gy) using a IMRT technique, Deutsch et al. [30] showed in a retrospective comparative analysis between EBRT vs. brachytherapy boost that the biochemical control remained significantly better in the brachytherapy group (3 $\times 7$ Gy). Whatever, it remains difficult to compare EBRT with brachytherapy boost techniques, because the dose delivered by brachytherapy will be always higher compare to the same prescribed dose delivered by EBRT (overdose volumes). This fact introduces an initial bias, which complicates the comparison between the two boost 
Table 2. Dosimetric data and equivalent doses at 2 Gy for CTV (EQD2 $\alpha / \beta 1.5$ Gy) and organs at risk (EQD2 $\alpha / \beta 3$ Gy) according to the treatment group

\begin{tabular}{|c|c|c|c|c|c|c|c|c|}
\hline \multirow{2}{*}{\multicolumn{2}{|c|}{ Data }} & \multicolumn{2}{|c|}{3 fractions (G1) } & \multicolumn{2}{|c|}{2 fractions (G2) } & \multicolumn{2}{|c|}{1 fraction (G3) } & \multirow[t]{2}{*}{$p$ value } \\
\hline & & Average & (Min-Max) & Average & (Min-Max) & Average & (Min-Max) & \\
\hline \multicolumn{2}{|c|}{ CTV (cc) } & 36 & $(16-60)$ & 33 & $(18-54)$ & 32 & $(11-77)$ & 0.088 \\
\hline \multirow[t]{2}{*}{$D_{90}$} & $\%$ & 114 & $(81-138)$ & 111 & $(95-144)$ & 105 & $(78-113)$ & $<0.001$ \\
\hline & EQD2 (Gy) & 49 & $(26-69)$ & 66 & $(49-107)$ & 68 & $(39-78)$ & $<0.001$ \\
\hline \multirow[t]{2}{*}{$\mathrm{D}_{100}$} & $\%$ & 84 & $(36-100)$ & 78 & $(54-98)$ & 78 & $(43-100)$ & 0.032 \\
\hline & EQD2 (Gy) & 28 & $(7-39)$ & 34 & $(18-52)$ & 39 & $(13-62)$ & 0.012 \\
\hline \multirow{2}{*}{$V_{100}$} & $\%$ & 97 & $(83-100)$ & 96 & $(88-99)$ & 95 & $(79-100)$ & 0.013 \\
\hline & $\mathrm{CC}$ & 35 & $(30-36)$ & 32 & $(29-33)$ & 30.4 & $(25-36)$ & 0.009 \\
\hline \multirow[t]{2}{*}{$V_{150}$} & $\%$ & 45 & $(16-81)$ & 36 & $(25-36)$ & 27 & $(18-39)$ & $<0.001$ \\
\hline & $\mathrm{CC}$ & 16 & $(6-29)$ & 12 & $(8-20)$ & 8.6 & $(6-12)$ & $<0.001$ \\
\hline \multirow[t]{2}{*}{$V_{200}$} & $\%$ & 16 & $(5-33)$ & 12 & $(8-19)$ & 9 & $(6-15)$ & $<0.001$ \\
\hline & $\mathrm{CC}$ & 6 & $(2-12)$ & 4 & $(3-6)$ & 3 & $(2-5)$ & $<0.001$ \\
\hline \multicolumn{2}{|l|}{$\mathrm{DHI}$} & 54 & $(18-81)$ & 61 & $(38-72)$ & 69 & $(43-81)$ & $<0.001$ \\
\hline \multirow[t]{2}{*}{$D_{0.1 u}$} & $\%$ & 150 & $(113-211)$ & 124 & $(117-168)$ & 113 & $(107-120)$ & $<0.001$ \\
\hline & EQD2 (Gy) & 54 & $(40-67)$ & 50 & $(33-66)$ & 48 & $(33-61)$ & $<0.001$ \\
\hline \multirow[t]{2}{*}{$\mathrm{D}_{1 \mathrm{u}}$} & $\%$ & 114 & $(44-153)$ & 101 & $(11-132)$ & 99 & $(10-112)$ & $<0.001$ \\
\hline & EQD2 (Gy) & 44 & $(32-53)$ & 39 & $(28-57)$ & 37 & $(28-50)$ & $<0.001$ \\
\hline \multirow[t]{2}{*}{$\mathrm{D}_{10 \mathrm{u}}$} & $\%$ & 141 & $(109-209)$ & 118 & $(112-146)$ & 112 & $(105-115)$ & $<0.001$ \\
\hline & EQD2 (Gy) & 52 & $(38-65)$ & 47 & $(31-63)$ & 46 & $(30-58)$ & $<0.001$ \\
\hline \multirow[t]{2}{*}{$D_{30 u}$} & $\%$ & 130 & $(105-183)$ & 114 & $(68-138)$ & 107 & $(44-111)$ & $<0.001$ \\
\hline & EQD2 (Gy) & 49 & $(33-59)$ & 44 & $(34-59)$ & 42 & $(32-52)$ & $<0.001$ \\
\hline \multirow[t]{2}{*}{$D_{0.1 r}$} & $\%$ & 102 & (91-123) & 94 & $(74-118)$ & 87 & $(53-99)$ & $<0.001$ \\
\hline & EQD2 (Gy) & 40 & $(25-47)$ & 38 & $(30-50)$ & 36 & $(29-44)$ & 0.005 \\
\hline \multirow[t]{2}{*}{$D_{1 r}$} & $\%$ & 84 & $(68-101)$ & 77 & $(53-91)$ & 70 & $(31-87)$ & $<0.001$ \\
\hline & EQD2 (Gy) & 32 & $(14-41)$ & 31 & $(22-39)$ & 29 & $(22-37)$ & 0.008 \\
\hline \multirow[t]{2}{*}{$D_{2 r}$} & $\%$ & 76 & $(57-93)$ & 68 & $(46-83)$ & 62 & $(24-80)$ & $<0.001$ \\
\hline & EQD2 (Gy) & 28 & $(11-38)$ & 27 & $(20-35)$ & 26 & $(18-33)$ & 0.034 \\
\hline
\end{tabular}

CTV - clinical target volume (prostate), $D_{90}$-dose delivered to $90 \%$ of CTV expressed in percentage and in EQD2 with $\alpha / \beta 1.5$ Gy, $D_{100}-$ dose delivered to $100 \%$ of CTV expressed in percentage and in equivalence of the dose at 2 Gy per fraction (EQD2) with $\alpha / \beta 1.5 \mathrm{~Gy}, V_{100}-C T V$ receiving $100 \%$ of the prescribed dose expressed in percentage and in cubic centimetres, $V_{150}$ - CTV receiving $150 \%$ of the prescribed dose expressed in percentage and in cubic centimetres, $V_{200}-C T V$ receiving $200 \%$ of the prescribed dose expressed in percentage and in cubic centimetres, DHI - Dose Homogeneity Index, $D_{0.14}-$ dose delivered to $0.1 \mathrm{cc}$ of the urethral volume expressed in percentage of the prescribed dose and in EQD2 with $\alpha / \beta 3 \mathrm{~Gy}, D_{1 u}$-dose delivered to $1 \mathrm{cc}$ of the urethral volume expressed in percentage of the prescribed dose and in EQD2 with $\alpha / \beta 3$ Gy, $D_{10 u}$ - dose delivered to $10 \%$ of urethral volume expressed in percentage of the prescribed dose and in EQD2 with $\alpha / \beta 3 \mathrm{~Gy}, D_{30 u}$ - dose delivered to $30 \%$ of urethral volume expressed in percentage of the prescribed dose and in EQD2 with $\alpha / \beta 3$ Gy, $D_{01 r}-$ dose delivered to $0.1 \mathrm{cc}$ of the rectal volume expressed in percentage of the prescribed dose and in EQD2 with $\alpha / \beta 3 \mathrm{~Gy}, D_{1 r}$-dose delivered to 1 cc of the rectal volume expressed in percentage of the prescribed dose and in EQD2 with $\alpha / \beta 3 \mathrm{~Gy}, D_{2 r}$-dose delivered to $2 \mathrm{cc}$ of the rectal volume expressed in percentage of the prescribed dose and in EQD2 with $\alpha / \beta 3$ Gy

techniques. Taking all together, the presented studies bring consistent data to consider brachytherapy boost as an attractive concept.

Currently, there is no standard fractionation scheme, and numerous protocols have been tested with various numbers of fractions (ranged from 1 to 5) and dose per fraction (ranged from 4.75 to $15 \mathrm{~Gy}$ ) [13]. It appears more comfortable for both patient and medical staff to reduce as far as possible the number of brachytherapy fractions respecting a similar biological effect, and clinical outcome in terms of toxicity and efficacy. In this filed, we started our brachytherapy boost program using 3 fractions in 2 days $(3 \times 6 \mathrm{~Gy})$, while one fraction of 14 Gy became our standard protocol allowing to implant the needles, treat the patient and remove all the materials in the same day (one day procedure). According to acute GI and GU toxicities, Hoskin et al. [29] reported in a phase III randomised trial that HDRB boost technique could significantly reduce GI toxicity with equivalent GU complication rates compared to those observed with EBRT. Regarding acute GI and GU toxicities, we reported mainly grade 1 complications and especially no significant differences between the 3 fractionations schemes. Furthermore, the dose distribution improved according to the learning curve, although the dose constraints for OARs were more restrictive. Our results in terms of EQD2 for urethra and rectum (Boost EQD2 + EBRT 46 Gy) respect the recently published GEC-ESTRO recommendations [15], which propose for urethra $\mathrm{D}_{0.1 \mathrm{cc}}$ and $\mathrm{D}_{10} \leq 120 \mathrm{~Gy}$ EQD2 with $\mathrm{D}_{30} \leq 105$ Gy EQD2, and for rectum $\mathrm{D}_{2 \mathrm{cc}} \leq 75$ Gy EQD2. 
Table 3. Analysis of genito-urinary (GU) and gastro-intestinal (GI) complications observed at 1 and 6 months after HDRB boost regarding to the complication grade (CTCV3.0) and the different fractionation schemes

\begin{tabular}{|c|c|c|c|c|}
\hline \multirow[t]{2}{*}{ Complications } & \multicolumn{2}{|c|}{ GU } & \multicolumn{2}{|c|}{ GI } \\
\hline & 1 month (\%) & 6 months (\%) & 1 month (\%) & 6 months (\%) \\
\hline \multicolumn{5}{|l|}{ Grades } \\
\hline 0 & 61 & 69 & 74 & 77 \\
\hline 1 & 33 & 31 & 26 & 22 \\
\hline 2 & 5 & 0 & 0 & 1 \\
\hline 3 & 0 & 0 & 0 & 0 \\
\hline 4 & 1 & 0 & 0 & 0 \\
\hline \multicolumn{5}{|l|}{ Fract. schemes } \\
\hline G1 (18 Gy/3 f/2 d) & $22^{*}$ & 33 & 23 & 45 \\
\hline G2 (18 Gy/2f/2 d) & 51 & 63 & 30 & 50 \\
\hline G3 (14 Gy/1 f/1 d) & 27 & 4 & 47 & 5 \\
\hline$p$ value & 0.41 & 0.49 & 0.07 & 0.34 \\
\hline
\end{tabular}

*Percentages of complication among patients who developed GU and/or GI complications

GU - genito-urinary complications, GI - gastro-intestinal complications, Fract. schemes - fractionation schemes

Currently, the right fractionation remains under debate, but following strict implantation rules [31], there is a strong rational to propose a prostate cancer HDRB boost using a single fraction, providing similar results in terms of acute toxicity, and more comfortable procedure for patient and less time consuming for medical staff. However, longer follow-up is needed to confirm that single fraction of HDRB used as boost will give consistent results in terms of clinical outcome.

\section{Conclusions}

The right fractionation remains under discussion, but prostate cancer HDRB boost using a single fraction (providing similar results in terms of acute toxicity) is more comfortable for the patient, less time consuming for the medical staff.

\section{Disclosure}

Authors report no conflict of interest.

\section{References}

1. Grenabo Bergdahl A, Holmberg E, Moss S et al. Incidence of Prostate Cancer After Termination of Screening in a Population-based Randomised Screening Trial. Eur Urol 2013; 64: 703-709.

2. Bolla M, Gonzalez D, Warde P et al. Improved survival in patients with locally advanced prostate cancer treated with radiotherapy and goserelin. N Engl J Med 1997; 337: 295-300.

3. Hanks GE, Pajak TF, Porter A et al. Phase III trial of longterm adjuvant androgen deprivation after neoadjuvant hormonal cytoreduction and radiotherapy in locally advanced carcinoma of the prostate: The Radiation Therapy Oncology Group protocol 92-02. J Clin Oncol 2003; 21: 3972-3978.

4. Pollack A, Zagars GK, Smith LG et al. Preliminary results of a randomized radiotherapy dose-escalation study comparing 70 Gy with 78 Gy for prostate cancer. J Clin Oncol 2000; 18: 3904-3911.

5. Shipley WU, Verhey LJ, Munzenrider JE et al. Advanced prostate cancer: the results of a randomized comparative trial of high dose irradiation boosting with conformal protons compared with conventional dose irradiation using photons alone. Int J Radiat Oncol Biol Phys 1995; 32: 3-12.

6. Peeters ST, Heemsbergen WD, Koper PC et al. Dose-response in radiotherapy for localized prostate cancer: results of the Dutch multicenter randomized phase III trial comparing 8 Gy of radiotherapy with 78 Gy. J Clin Oncol 2006; 24: 1990-1996.

7. Dearnaley DP, Sydes MR, Graham JD et al. Escalated-dose versus standard-dose conformal radiotherapy in prostate cancer: first results from the MRC RT01 randomized controlled trial. Lancet Oncol 2007; 8: 475-487.

8. Beckendorf V, Guerif S, Le Prisé E et al. 70 Gy versus 80 Gy in localized prostate cancer: 5-year results of GETUG 06 randomized trial. Int J Radiat Oncol Biol Phys 2011; 80: 1056-1063.

9. Kuban DA, Tucker SL, Dong L et al. Long-term results of the M. D. Anderson randomized dose-escalation trial for prostate cancer. Int J Radiat Oncol Biol Phys 2008; 70: 67-74.

10. Zelefsky MJ, Levin EJ, Hunt M et al. Incidence of late rectal and urinary toxicities after three-dimensional conformal radiotherapy and intensity-modulated radiotherapy for localized prostate cancer. Int J Radiat Oncol Biol Phys 2008; 70: 1124-1129.

11. Pollack A, Hanlon AL, Horwitz EM et al. Dosimetry and preliminary acute toxicity in the first 100 men treated for prostate cancer on a randomized hypofractionation dose escalation trial. Int J Radiat Oncol Biol Phys 2006; 64: 518-526.

12. Arcangeli G, Fowler J, Gomellini S et al. Acute and late toxicity in a randomized trial of conventional versus hypofractionated three-dimensional conformal radiotherapy for prostate cancer. Int J Radiat Oncol Biol Phys 2011; 79: 1013-1021.

13. Yamada Y, Rogers L, Demanes DJ et al. American Brachytherapy Society. American Brachytherapy Society consensus guidelines for high-dose-rate prostate brachytherapy. Brachytherapy 2012; 11: 20-32.

14. Roach M 3rd, Marquez C, Yuo HS et al. Predicting the risk of lymph node involvement using the pre-treatment prostate specific antigen and Gleason score in men with clinically localized prostate cancer. Int J Radiat Oncol Biol Phys 1994; 28: 33-37.

15. Hoskin PJ, Colombo A, Henry A, et al. GEC/ESTRO recommendations on high dose rate afterloading brachytherapy for 
localised prostate cancer: An update. Radiother Oncol 2013; 107: 325-332.

16. Fowler JF. The radiobiology of prostate cancer including new aspects of fractionated radiotherapy. Acta Oncol 2005; 44: 265-276.

17. Simnor T, Li S, Lowe G et al. Justification for inter-fraction correction of catheter movement in fractionated high doserate brachytherapy treatment of prostate cancer. Radiother Oncol 2009; 93: 253-258.

18. Common Terminology Criteria for Adverse Event (CTCAE v3.0). http://ctep.cancer.gov/protocolDevelopment/ electronic_applications/docs/ctcaev3.pdf. Access on line 03/15/2013.

19. Roach M III, Hanks G, Thames H Jr. et al. Defining biochemical failure following radiotherapy with or without hormonal therapy in men with clinically localized prostate cancer: Recommendations of the RTOG-ASTRO Phoenix Consensus Conference. Int J Radiat Oncol Biol Phys 2006; 65: 965-974.

20. D'Amico AV, Whittington R, Malkowicz SB et al. Biochemical outcome after radical prostatectomy, external beam radiation therapy, or interstitial radiation therapy for clinically localized prostate cancer. JAMA 1998; 280: 969-974.

21. Viani GA, Stefano EJ, Afonso SL. Higher-than-conventional radiation doses in localized prostate cancer treatment: a meta-analysis of randomized, controlled trials. Int J Radiat Oncol Biol Phys 2009; 74: 1405-1418.

22. Martinez AA, Gonzalez J, Ye $\mathrm{H}$ et al. Dose escalation improves cancer-related events at 10 years for intermediate- and high-risk prostate cancer patients treated with hypofractionated high-dose-rate boost and external beam radiotherapy. Int J Radiat Oncol Biol Phys 2011; 79: 363-370.

23. Prada PJ, Mendez L, Fernández J et al. Long-term biochemical results after high-dose-rate intensity modulated brachytherapy with external beam radiotherapy for high risk prostate cancer. Radiat Oncol. 2012; 7: 31. doi: 10.1186/1748-717X-7-31.

24. Hannoun-Levi JM, Chand-Fouche ME, Dejean C et al. Dose gradient impact on equivalent dose at $2 \mathrm{~Gy}$ for high dose rate interstitial brachytherapy. J Contemp Brachytherapy 2012; 4: 14-20.

25. Skowronek J. Low-dose-rate or high-dose-rate brachytherapy in treatment of prostate cancer - between options. J Contemp Brachytherapy 2013; 5: 33-41.

26. Kestin LL, Martinez AA, Stromberg JS et al. Matched-pair analysis of conformal high-dose-rate brachytherapy boost versus external-beam radiation therapy alone for locally advanced prostate cancer. J Clin Oncol 2000; 18: 2869-2880.

27. Sathya JR, Davis IR, Julian JA et al. Randomized trial comparing iridium implant plus external-beam radiation therapy with external-beam radiation therapy alone in node-negative locally advanced cancer of the prostate. J Clin Oncol 2005; 23: 1192-1199.

28. Hoskin PJ, Rojas AM, Bownes PJ et al. Randomised trial of external beam radiotherapy alone or combined with highdose-rate brachytherapy boost for localised prostate cancer. Radiother Oncol 2012; 10: 217-222.

29. Hoskin PJ, Motohashi K, Bownes P et al. High dose rate brachytherapy in combination with external beam radiotherapy in the radical treatment of prostate cancer: initial results of a randomised phase three trial. Radiother Oncol 2007; 84: 114-120.

30. Deutsch I, Zelefsky MJ, Zhang Z et al. Comparison of PSA relapse-free survival in patients treated with ultra-high-dose IMRT versus combination HDR brachytherapy and IMRT. Brachytherapy 2010; 9: 313-318.

31. Wojcieszek P, Białas B. Prostate cancer brachytherapy: guidelines overview. J Contemp Brachytherapy 2012; 4: 116-120. 